\title{
GENERALIZED NEUMANN AND KAPTEYN EXPANSIONS
}

\author{
HAROLD EXTON \\ "Nyuggel", Lunabister \\ Dunrossness, Shetland ZE2 9JH \\ United Kingdom
}

(Received December, 1994; Revised June, 1995)

\begin{abstract}
Certain formal series of a most general nature are specialized so as to deduce expansions in terms of a class of generalized hypergeometric functions. These series generalize the Neumann and Kapteyn series in the theory of Bessel functions, and their convergence is investigated. An example of a succinct expansion is also given.
\end{abstract}

Key words: Neumann, Kapteyn.

AMS (MOS) subject classifications: $33 \mathrm{C} 20,33 \mathrm{C} 10$.

\section{Introduction}

Certain formal series which generalize the Neumann and Kapteyn series have quite recently been introduced by Exton [2]. These results are embodied in Lemmas 1 and 2 of the same study and are now quoted for convenience.

Lemma 1: If $C(\mu)$ is an arbitrary function of $\mu$ and if

$$
X_{\nu}=\sum_{r=0}^{\infty} \frac{(-1)^{r} C\left(\frac{1}{2} \nu+r\right)}{r ! \Gamma(\nu+r+1)}
$$

then we have the formal result

$$
C\left(\frac{1}{2} \nu\right)=\sum_{k=0}^{\infty} \frac{(\nu+2 k) \Gamma(\nu+k)}{k !} X_{\nu+2 k}
$$

Lemma 2: If $C(\mu)$ is an arbitrary function of $\mu$ and if

$$
Y_{\nu}=\sum_{r=0}^{\infty} \frac{(-1)^{r} C\left(\frac{1}{2} \nu+r\right) \nu^{\nu+2 r}}{r ! \Gamma(\nu+r+1)}
$$

then we have the formal result

$$
C\left(\frac{1}{2} \nu\right)=\nu^{2} \sum_{k=0}^{\infty} \frac{\Gamma(\nu+k)}{(\nu+2 k)^{\nu+1} k !} Y_{\nu+2 k}
$$

In Lemma 1, we put 


$$
C(\mu)=\frac{\Gamma\left(a_{1}+\mu\right) \ldots \Gamma\left(a_{n}+\mu\right)}{\Gamma\left(b_{1}+\mu\right) \ldots \Gamma\left(b_{n}+\mu\right)}\left(\frac{1}{2} z\right)^{2 \mu}
$$

and $X_{\nu}={ }_{n} X_{\nu}(z)={ }_{n} X_{\nu}\left(a_{1}, \ldots, a_{n} ; b_{1}, \ldots, b_{n} ; z\right)={ }_{n} X_{\nu}((a) ;(b) ; z)$

$$
=\sum_{r=0}^{\infty} \frac{(-1)^{r} \Gamma\left(a_{1}+\frac{1}{2} \nu+r\right) \ldots \Gamma\left(a_{n}+\frac{1}{2} \nu+r\right)\left(\frac{1}{2} z\right)^{\nu+2 r}}{\Gamma\left(b_{1}+\frac{1}{2} \nu+r\right) \ldots \Gamma\left(b_{n}+\frac{1}{2} \nu+r\right) \Gamma(\nu+1+r) r !} .
$$

The function given in (1.1) can be expressed as a generalized hypergeometric function as follows:

$$
\begin{gathered}
{ }_{n} X_{\nu}((a) ;(b) ; z)=\frac{\Gamma\left(a_{1}+\frac{1}{2} \nu\right) \ldots \Gamma\left(a_{n}+\frac{1}{2} \nu\right)\left(\frac{1}{2} z\right)^{\nu}}{\Gamma\left(b_{1}+\frac{1}{2} \gamma\right) \ldots \Gamma\left(b_{n}+\frac{1}{2} \nu\right) \Gamma(\nu+1)} \\
\cdot{ }_{n} F_{n+1}\left[\begin{array}{c}
a_{1}+\frac{1}{2} \nu, \ldots, a_{n}+\frac{1}{2} \nu ; \\
b_{1}+\frac{1}{2} \nu, \ldots, b_{n}+\frac{1}{2} \nu, 1+\nu ;
\end{array}-\frac{1}{4} z^{2}\right] .
\end{gathered}
$$

If $n=0$, this function reduces to the Bessel function $J_{\nu}(z)$. The series on the right of (1.2) converges absolutely and uniformly for all finite values of $|z|$. For a comprehensive treatment of generalized hypergeometric series, the reader should consult Slater [3] for example.

\section{A Generating Function and Recurrence Relations}

The function

$$
V=V((a) ;(b))=\sum_{r=0}^{\infty} \frac{\Gamma\left(a_{1}+\frac{1}{2} r\right) \ldots \Gamma\left(a_{n}+\frac{1}{2} r\right)}{\Gamma\left(b_{1}+\frac{1}{2} r\right) \ldots \Gamma\left(b_{n}+\frac{1}{2} r\right) r !}\left[z\left(t-t^{-1}\right) / 2\right]^{r}
$$

is arranged in powers of $t$. As a simple consequence of the binomial theorem, we have

$$
V=\sum_{p, q=0}^{\infty} \frac{\Gamma\left(a_{1}+\frac{1}{2} p+\frac{1}{2} q\right) \ldots \Gamma\left(a_{n}+\frac{1}{2} p+\frac{1}{2} q\right)(-1)^{p}\left(\frac{1}{2} z\right)^{p+q} t^{q-p}}{\Gamma\left(b_{1}+\frac{1}{2} p+\frac{1}{2} q\right) \ldots \Gamma\left(b_{n}+\frac{1}{2} p+\frac{1}{2} q\right) p ! q !}
$$

Put $q=m+p$ and rearrange, so that

$$
\begin{gathered}
V=\sum_{p=0}^{\infty} \frac{(-1)^{p}\left(\frac{1}{2} z\right)^{p}}{p !} \sum_{m}^{\infty} \frac{\Gamma\left(a_{1}+\frac{1}{2} m+p\right) \ldots \Gamma\left(a_{n}+\frac{1}{2} m+p\right)\left(\frac{1}{2} z\right)^{m+p}}{\Gamma\left(b_{1}+\frac{1}{2} m+p\right) \ldots \Gamma\left(b_{n}+\frac{1}{2} m+p\right)(m+p) !} t^{m} \\
=\sum_{m=-\infty}^{\infty} t^{m} \sum_{p=0}^{\infty} \frac{(-1)^{p} \Gamma\left(a_{1}+\frac{1}{2} m+p\right) \ldots \Gamma\left(a_{n}+\frac{1}{2} m+p\right)\left(\frac{1}{2} z\right)^{m+2 p}}{\Gamma\left(b_{1}+\frac{1}{2} m+p\right) \ldots \Gamma\left(b_{n}+\frac{1}{2} m+p\right)(m+p) ! p !} \\
=\sum_{m}^{\infty} t^{m}{ }_{n} X_{m}((a) ;(b) ; z) .
\end{gathered}
$$

As in the case of the Bessel coefficients, it is clear that

$$
{ }_{n} X_{-m}((a) ;(b) ; z)=(-1)^{m}{ }_{n} X_{m}((a) ;(b) ; z) .
$$

The generating function (2.1) readily yields recurrence relations for the function ${ }_{n} X_{m}((a) ;(b) ; z)$ which are exactly analogous to those which apply to the Bessel coefficient $J_{m}(z)$. 
Take partial derivatives with respect to $t$ of both members of (2.1). This gives

$$
\frac{1}{2} z\left(1+t^{-2}\right) V\left(\left(a+\frac{1}{2}\right) ;\left(b+\frac{1}{2}\right)\right)=\sum_{m}^{\infty} m t^{m-1}{ }_{n} X_{m}((a) ;(b) ; z) .
$$

On equating the coefficients of successive powers of $t$ to zero, it follows from (2.2) that

$$
\begin{gathered}
{ }_{n} X_{m-1}\left(\left(a+\frac{1}{2}\right) ;\left(b+\frac{1}{2}\right) ; z\right)+{ }_{n} X_{m+1}\left(\left(a+\frac{1}{2}\right) ;\left(b+\frac{1}{2}\right) ; z\right) \\
=2 m_{n} X_{m}((a) ;(b) ; z) / z
\end{gathered}
$$

Similarly, taking partial derivatives of (2.1) with respect to $z$, it is found that

$$
\frac{1}{2}\left(t-t^{-1}\right) V\left(\left(a+\frac{1}{2}\right) ;\left(b+\frac{1}{2}\right)\right)=\sum_{m}^{\infty} t^{m}{ }_{n} X_{m}^{\prime}((a) ;(b) ; z)
$$

and we have

$$
{ }_{n} X_{m-1}\left(\left(a+\frac{1}{2}\right) ;\left(b+\frac{1}{2}\right) ; z\right)-{ }_{n} X_{m+1}\left(\left(a+\frac{1}{2}\right) ;\left(b+\frac{1}{2}\right) ; z\right)={ }_{n} X_{m}^{\prime}((a) ;(b) ; z),
$$

where the primes denote differentiations with respect to $z$. On adding and subtracting (2.3) and $(2.4)$, we obtain

$$
z_{n} X_{m}^{\prime}((a) ;(b) ; z)+m_{n} X_{m}((a) ;(b) ; z)=z_{n} X_{m-1}\left(\left(a+\frac{1}{2}\right) ;\left(b+\frac{1}{2}\right) ; z\right)
$$

and

$$
z_{n} X_{m}^{\prime}((a) ;(b) ; z)-m_{n} X_{m}((a) ;(b) ; z)=-z_{n} X_{m+1}\left(\left(a+\frac{1}{2}\right) ;\left(b+\frac{1}{2}\right) ; z\right) \text {. }
$$

The expressions (2.5) and (2.6) may respectively be written in the form

$$
\begin{gathered}
\frac{d}{d z}\left[z_{n}^{m} X_{m}((a) ;(b) ; z)\right]=z^{m}{ }_{n} X_{m-1}\left(\left(a+\frac{1}{2}\right) ;\left(b+\frac{1}{2}\right) ; z\right) \\
\frac{d}{d z}\left[z^{-m} X_{m}((a) ;(b) ; z)\right]=-z^{-m} X_{m+1}\left(\left(a+\frac{1}{2}\right) ;\left(b+\frac{1}{2}\right) ; z\right) .
\end{gathered}
$$

Replace $m$ by $m-1$ in $(2.8)$ and eliminate ${ }_{n} X_{m-1}\left(\left(a+\frac{1}{2}\right) ;\left(b+\frac{1}{2}\right) ; z\right)$ between the result and (2.7):

$$
\frac{d}{z}\left\{z^{1-2 m} \frac{d}{d z}\left[z^{m}{ }_{n} X_{m}((a) ;(b) ; z)\right]\right\}=-z^{1-m} X_{m}((a+1) ;(b+1) ; z) .
$$

That is,

$$
\begin{gathered}
\frac{d}{d z}\left[z^{1-m} \frac{d}{d z} X_{m}((a) ;(b) ; z)+m z_{n}^{-m} X_{m}((a) ;(b) ; z)\right] \\
=-z^{1-m} X_{m}((a+1) ;(b+1) ; z)
\end{gathered}
$$

which on expansion becomes

$$
\begin{gathered}
z^{2} \frac{d^{2}}{d z^{2}}{ }_{n} X_{m}((a) ;(b) ; z)+z \frac{d}{d z}{ }_{n} X_{m}((a) ;(b) ; z)-m_{n}^{2} X_{m}((a) ;(b) ; z) \\
+z^{2}{ }_{n} X_{m}((a+1) ;(b+1) ; z)=0 .
\end{gathered}
$$

This differentio-difference equation (2.9) corresponds exactly with Bessel's equation to which it reduces when $n=0$, see Watson [4]. 


\section{Expansions of Neumann Type}

A result, formal at first, is obtained from Lemma 1 of Exton [2] with the forms of $C(\mu)$ and ${ }_{n} X_{\nu}$ used in (1.1). This is

$$
\frac{\Gamma\left(a_{1}+\frac{1}{2} \nu\right) \ldots \Gamma\left(a_{n}+\frac{1}{2} \nu\right)}{\Gamma\left(b_{1}+\frac{1}{2} \nu\right) \ldots \Gamma\left(b_{n}+\frac{1}{2} \nu\right)}\left(\frac{1}{2} z\right)^{\nu}=\sum_{k=0}^{\infty} \frac{(\nu+2 k) \Gamma(\nu+k)}{k !} \cdot{ }_{n} X_{\nu+2 k}((a) ;(b) ; z)
$$

where the convergence of the series on the right of (3.1) remains to be established.

For large values of $\nu$,

$$
\prod_{j=1}^{n}\left[\Gamma\left(a_{j}+\frac{1}{2} \nu+r\right) / \Gamma\left(b_{j}+\frac{1}{2} \nu+r\right)\right] \sim\left(\frac{1}{2} \nu\right)^{d}
$$

where, for convenience, we have put $d=\sum_{j=1}^{n}\left(a_{j}-b_{j}\right)$. It then follows that for large values of $\nu$,

$$
\left.{ }_{n} X_{\nu}(a) ;(b) ; z\right) \sim\left(\frac{1}{2} \nu\right)^{d} J_{\nu}(z) .
$$

From an inequality given by Watson [4], page 44, it then follows that, for large values of $k$

$$
{ }_{n} X_{\nu+2 k}((a) ;(b) ; z) \sim \frac{k^{d}\left(\frac{1}{2} z\right)^{\nu+2 k}}{\Gamma(1+\nu+2 k)}(1+\theta),
$$

where $|\theta|<\exp \left[|z|^{2} /\left(4\left|\nu_{0}+1\right|\right)\right]-1$ and where $\nu_{0}$ is the smallest of the numbers $|\nu+1|$, $|\nu+2|,|\nu+3|, \ldots$ The general term of the series (3.1) is given by

$$
T_{k}=\frac{(\nu+2 k) \Gamma(\nu+k)}{k !}{ }_{n} X_{\nu+2 k}((a) ;(b) ; z) \sim \frac{(\nu+2 k) \Gamma(\nu+k)\left(\frac{1}{2} z\right)^{\nu+2 k}}{k ! \Gamma(1+\nu+2 k)}(1+\theta) .
$$

After a little algebra, it may be found that, for sufficiently large values of $k$ that

$$
T_{k+1} / T_{k} \sim \frac{(\nu+k)}{(\nu+2 k)(\nu+2 k+1)(k+1)}[(k+1) / k]^{d}\left(\frac{1}{2} z\right)^{\nu+2}
$$

and $\lim _{k \rightarrow \infty}\left(T_{k+1} / T_{k}\right)=0$, so that the series (3.1) converges absolutely and uniformly within any bounded region of the $z$-plane. A number of expansions in series of the functions ${ }_{n} X_{\nu+2 k}$ may thus be deduced from (3.1). For example, we give an expansion of a function of a similar type. Now

$$
\begin{gathered}
\left(\frac{1}{2} k z\right)^{\mu-\nu} X_{\nu}((a) ;(b) ; k z) \\
=k^{\mu} \sum_{m=0}^{\infty} \frac{(-1)^{m} \Gamma\left(a_{1}+\frac{1}{2} \nu+m\right) \ldots \Gamma\left(a_{g}+\frac{1}{2} \nu+m\right)\left(\frac{1}{2} z\right)^{\mu+2 m} k^{2 m}}{\Gamma(\nu+m+1) \Gamma\left(b_{1}+\frac{1}{2} \nu+m\right) \ldots \Gamma\left(b_{g}+\frac{1}{2} \nu+m\right) m !} .
\end{gathered}
$$

The power of $z$ on the right, $\left(\frac{1}{2} z\right)^{\mu+2 m}$, is replaced by its expansion (3.1), and we have

$$
\begin{gathered}
\left(\frac{1}{2} k z\right)^{\mu-\nu} X_{\nu}((a) ;(b) ; k z) \\
=k^{\mu} \sum_{m=0}^{\infty} \frac{(-1)^{m} \Gamma\left(a_{1}+\frac{1}{2} \nu+m\right) \ldots \Gamma\left(a_{g}+\frac{1}{2} \nu+m\right)}{\Gamma\left(b_{1}+\frac{1}{2} \nu+m\right) \ldots \Gamma\left(b_{g}+\frac{1}{2} \nu+m\right) \Gamma(\nu+m+1) m !}
\end{gathered}
$$




$$
\begin{gathered}
\cdot \frac{\Gamma\left(b_{1}^{\prime}+\frac{1}{2} \nu+m\right) \ldots \Gamma\left(b_{q}^{\prime}+\frac{1}{2} \mu+m\right) k^{2 m}}{\Gamma\left(a_{1}^{\prime}+\frac{1}{2} \mu+m\right) \ldots \Gamma\left(a_{q}^{\prime}+\frac{1}{2} \mu+m\right)} \\
\cdot \sum_{p=0}^{\infty} \frac{(\mu+2 m+2 p) \Gamma(\mu+2 m+p)}{p !}{ }_{q} X_{\mu+2 m+2 p}\left(\left(a^{\prime}\right) ;\left(b^{\prime}\right) ; z\right) .
\end{gathered}
$$

Since the series concerned are absolutely convergent, the right-hand member of (3.3) can be rearranged in the form

$$
\begin{gathered}
k^{\mu} \sum_{n=0}^{\infty}\left[\sum_{m=0}^{n} \frac{(-1)^{m} \Gamma(\mu+m+n) \Gamma\left(a_{1}+\frac{1}{2} \nu+m\right) \ldots \Gamma\left(a_{g}+\frac{1}{2} \nu+m\right)}{m !(n-m) ! \Gamma(\nu+m+1) \Gamma\left(b_{1}+\frac{1}{2} \nu+m\right) \ldots \Gamma\left(b_{g}+\frac{1}{2} \nu+m\right)}\right. \\
\left.\cdot \frac{\Gamma\left(b_{1}^{\prime}+\frac{1}{2} \mu+m\right) \ldots \Gamma\left(b_{q}^{\prime}+\frac{1}{2} \mu+m\right) k^{2 m}}{\Gamma\left(a_{1}^{\prime}+\frac{1}{2} \mu+m\right) \ldots \Gamma\left(a_{q}^{\prime}+\frac{1}{2} \mu+m\right)}\right](\mu+2 n)_{q} X_{\mu+2 n}\left(\left(a^{\prime}\right) ;\left(b^{\prime}\right) ; z\right) .
\end{gathered}
$$

The inner summation in $m$ can be interpreted as a generalized hypergeometric function, so that we have, finally

$$
\begin{gathered}
\left(\frac{1}{2} k z\right)^{\mu-\nu} X_{\nu}((a) ;(b) ; k z) \\
=\frac{\Gamma\left(a_{1}+\frac{1}{2} \nu\right) \ldots \Gamma\left(a_{g}+\frac{1}{2} \nu\right) \Gamma\left(b_{1}^{\prime}+\frac{1}{2} \mu\right) \ldots \Gamma\left(b_{q}^{\prime}+\frac{1}{2} \mu\right)}{\Gamma(\nu+1) \Gamma\left(b_{1}+\frac{1}{2} \nu\right) \ldots \Gamma\left(b_{g}+\frac{1}{2} \nu\right) \Gamma\left(a_{1}^{\prime}+\frac{1}{2} \mu\right) \ldots \Gamma\left(a_{q}^{\prime}+\frac{1}{2} \mu\right)} k^{\mu} \sum_{n=0}^{\infty} \frac{\Gamma(\mu+n)(\mu+2 n)}{n !} \\
\cdot{ }_{g+q+2} F_{g+q+1}\left[\begin{array}{c}
a_{1}+\frac{1}{2} \nu, \ldots, a_{g}+\frac{1}{2} \nu, b_{1}^{\prime}+\frac{1}{2} \mu, \ldots, b_{q}^{\prime}+\frac{1}{2} \mu, \mu+n,-n ; k^{2} \\
b_{1}+\frac{1}{2} \nu, \ldots, b_{g}+\frac{1}{2} \nu, a_{1}^{\prime}+\frac{1}{2} \mu, \ldots, a_{q}^{\prime}+\frac{1}{2} \mu, \nu+1 ;
\end{array}\right] \\
\cdot{ }_{q} X_{\mu+2 n}\left(\left(a^{\prime}\right) ;\left(b^{\prime}\right) ; z\right) .
\end{gathered}
$$

If the parameters and variable of the inner hypergeometric function of (3.4) can be so chosen that it is summable in a compact form, then a more elegant result follows in which only one summation is involved. It will be seen that if $g=q=0$, the formula (3) in Section 5.21 on page 140 of Watson [4] is recovered.

In (3.1), put $q=g, k=1, a_{i}^{\prime}=a_{i}+\frac{1}{2} \nu-\frac{1}{2} \mu$ and $b_{i}^{\prime}=b_{i}+\frac{1}{2} \nu-\frac{1}{2} \mu, 1 \leq i \leq g$, so that the inner hypergeometric function is reduced to a terminating function ${ }_{2} F_{1}$ of unit argument, summable by Vandermonde's theorem (Slater [3], page 243, for example). We then have the interesting result

$$
\begin{gathered}
\left(\frac{1}{2} z\right)^{\mu-\nu}{ }_{g} X_{\nu}((a) ;(b) ; z)=\sum_{n=0}^{\infty} \frac{\Gamma(\mu+n) \Gamma(\nu+1-\mu)(\mu+2 n)}{\Gamma(\nu+1-\mu-n) \Gamma(\nu+n+1) n !} \\
\cdot{ }_{g} X_{\mu+2 n}\left(\left(a+\frac{1}{2} \nu-\frac{1}{2} \mu\right) ;\left(b+\frac{1}{2} \nu-\frac{1}{2} \mu\right) ; z\right),
\end{gathered}
$$

which is essentially not more complicated than the original formula for Bessel functions obtained when $g=0$.

Other expressions of a similar character can be worked out using known summation formulae for the generalized hypergeometric function after suitable specialization of the disposable parameters. 


\section{Series of Kapteyn Type}

From Lemma 2 of Exton [2], quoted in Section 1 of this study, it is clear that with the same form of $C(\mu)$,

$$
Y_{\nu}={ }_{n} X_{\nu}((a) ;(b) ; \nu z)
$$

by comparison with (1.1). We then have the (initially formal) result

$$
\begin{gathered}
\frac{\Gamma\left(a_{1}+\frac{1}{2} \nu\right) \ldots \Gamma\left(a_{n}+\frac{1}{2} \nu\right)}{\Gamma\left(b_{1}+\frac{1}{2} \nu\right) \ldots \Gamma\left(b_{n}+\frac{1}{2} \nu\right)}\left(\frac{1}{2} z\right)^{\nu} \\
=\nu^{2} \sum_{k=0}^{\infty} \frac{\Gamma(\nu+k)}{(\nu+2 k)^{\nu+1} k !}{ }^{n} X_{\nu+2 k}((a) ;(b) ;(\nu+2 k) z) .
\end{gathered}
$$

If $n=0$, the expansion (4.1) becomes a well-known result in the theory of Kapteyn series, that is

$$
\left(\frac{1}{2} z\right)^{\nu}=\nu^{2} \sum_{k=0}^{\infty} \frac{\Gamma(\nu+k)}{(\nu+2 k)^{\nu+1} k !} J_{\nu+2 k}((\nu+2 k) z)
$$

see Watson [4], page 571.

The convergence of the series on the right of (4.1) must now be examined. Recall from (3.2) that for large values of $k$

$$
{ }_{n} X_{\nu+2 k}((a) ;(b) ;(\nu+2 k) z) \sim k^{d} J_{\nu+2 k}((\nu+2 k) z)
$$

where $d=\sum_{j=1}^{n}\left(a_{j}-b_{j}\right)$. We are then led to consider the convergence of the auxiliary series

$$
\sum_{k=0}^{\infty} \frac{\Gamma(\nu+k) k^{d}}{(\nu+2 k)^{\nu+1} k !}=\sum_{k=0}^{\infty} u_{k}
$$

The test (5), in effect Raabe's test, given in Section (12,2) page 40 of Bromwich [1], is now applied. It is easily seen that

$$
\begin{aligned}
u_{k} / u_{k+1} & =\frac{\Gamma(\nu+k) k^{d}(\nu+2 k+2)^{\nu+1}(k+1) !}{(\nu+2 k)^{\nu+1} k ! \Gamma(\nu+k+1)(k+1)^{d}} \\
& =1+(2-d) / k+O\left(1 / k^{2}\right) .
\end{aligned}
$$

By virtue of the test mentioned above, the series (4.2) converges if $\operatorname{Re}(2-d)>1$ and diverges is $\operatorname{Re}(2-d) \leq 1$. Hence, $(4.2)$ is convergent if $\operatorname{Re}(d)<1$.

In turn, for sufficiently large values of $k$, we now discuss the convergence of the series

$$
\sum_{k=0}^{\infty} \frac{\Gamma(\nu+k) k^{d}}{(\nu+2 k)^{\nu+1} k !} J_{\nu+2 k}((\nu+2 k) z) .
$$

When $z$ is real and $\nu=N, N=0,1,2, \ldots, J_{N+2 k}((N+2 k) x) \leq 1$, Watson [4], page 31. Hence, (4.3) converges with (4.2), and under these circumstances, the expansion

$$
\frac{\Gamma\left(a_{1}+\frac{1}{2} N\right) \ldots \Gamma\left(a_{n}+\frac{1}{2} N\right)}{\Gamma\left(b_{1}+\frac{1}{2} n\right) \ldots \Gamma\left(b_{n}+\frac{1}{2} n\right)}\left(\frac{1}{2} x\right)^{N}=N^{2} \sum_{k=0}^{\infty} \frac{\Gamma(N+k)}{(N+2 k)^{N+1} k !}
$$




$$
\cdot{ }_{n} X_{N+2 k}((a) ;(b) ;(N+2 k) x)
$$

converges absolutely and uniformly if $\operatorname{Re}(d)<1$, for all values of $x$.

For complex values of $\nu$ and $z$, the series on the right of (4.1) converges if $z$ lies within the domain $K$, that is the interior of the region for which

$$
\left|\frac{z \exp \sqrt{\left(1-z^{2}\right)}}{1+\sqrt{\left(1-z^{2}\right)}}\right|<1,
$$

provided that $\operatorname{Re}(d)<1$, see Watson [4], page 559. The series on the right of (4.1) also converges on the boundary of the region $K$ with the further proviso that for the points $z= \pm 1$, it is necessary that $\nu$ should be real (Watson [4], page 574).

As in the case of the generalized Neumann series, the corresponding generalization of the Kapteyn series can, in many cases, give rise to expansions in the form of double series. However, in the latter case, it is much less likely that a reduction to a single series can be brought about.

\section{References}

[1] Bromwich, T.J., The Theory of Infinite Series, Macmillan, London 1931.

[2] Exton, H., On certain series which generalize the Neumann and Kapteyn series, Riv. Mat. Univ. Parma 13 (1987), 275-278.

[3] Slater, L.J., Generalized Hypergeometric Functions, Cambridge University Press 1966.

[4] Watson, G.N., Bessel Functions, Cambridge University Press 1948. 


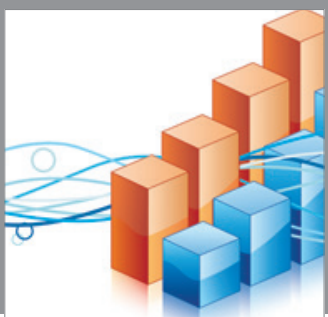

Advances in

Operations Research

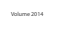

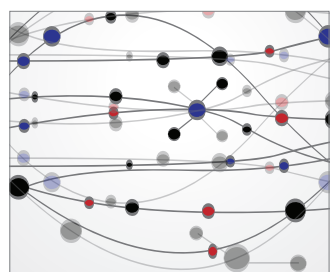

\section{The Scientific} World Journal
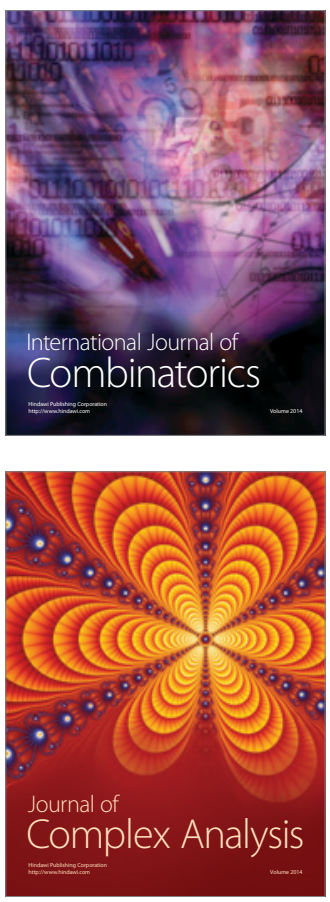

International Journal of

Mathematics and

Mathematical

Sciences
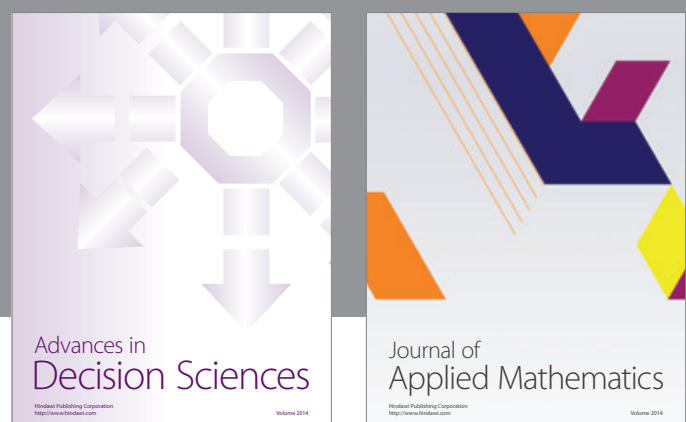

Journal of

Applied Mathematics
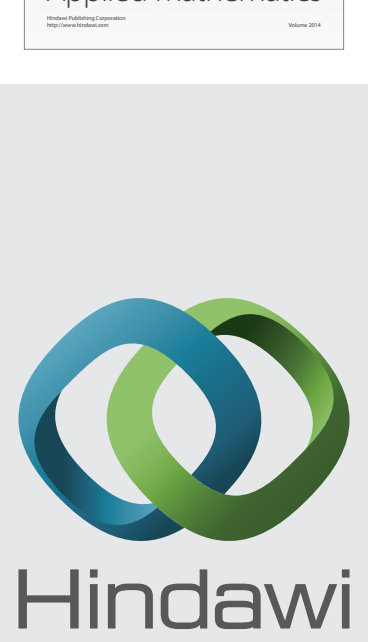

Submit your manuscripts at http://www.hindawi.com
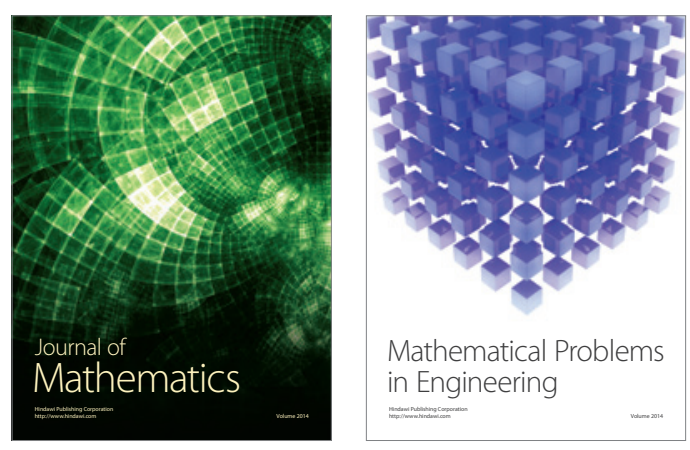

Mathematical Problems in Engineering
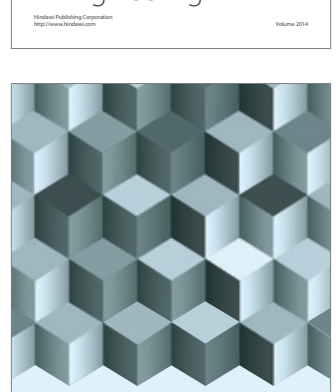

Journal of

Function Spaces
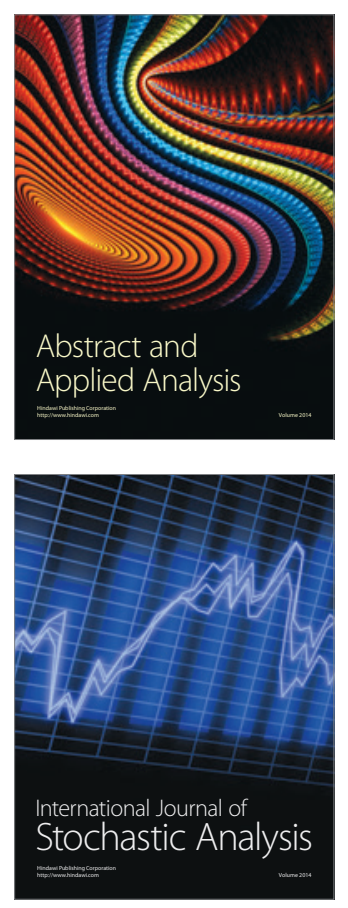

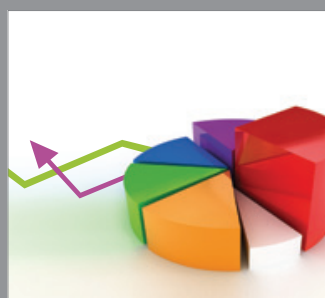

ournal of

Probability and Statistics

Promensencen
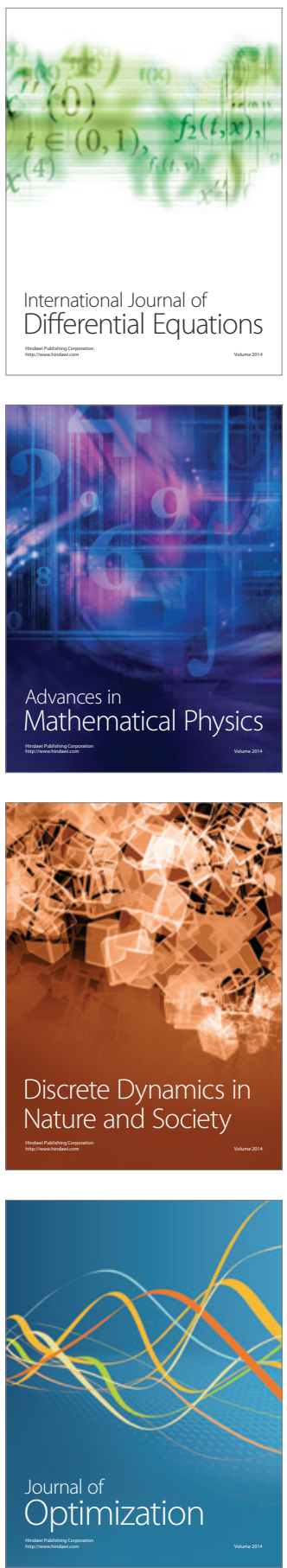